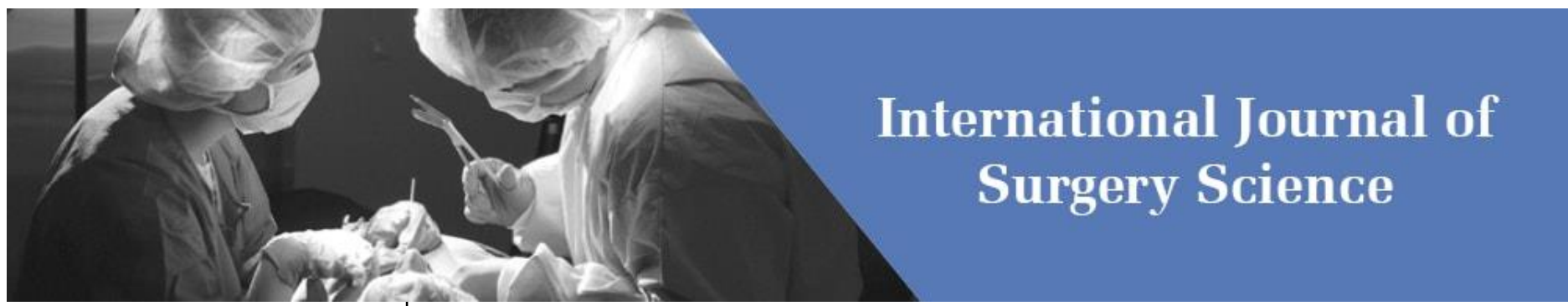

E-ISSN: 2616-3470

P-ISSN: 2616-3462

(C) Surgery Science

www.surgeryscience.com

2019; 3(3): 140-146

Received: 13-05-2019

Accepted: 18-06-2019

Dr. Labed Saadallah Abdulkareem Fallujah Teaching Hospital, Iraq

Dr. Akram N Dahel

Fallujah Teaching Hospital, Iraq

Dr. Mohammed Tafash Dagash

University of Anbar, Medical

College, Department of Surgery,

Iraq

\section{Acute mesenteric ischemia: An interventional study}

\author{
Dr. Labed Saadallah Abdulkareem, Dr. Akram N Dahel and Dr. Mohammed \\ Tafash Dagash
}

DOI: https://doi.org/10.33545/surgery.2019.v3.i3c.161

\section{Abstract}

From 1995 to 2005,50 patients with mesenteric ischemia diagnosed at laparotomy were studied in Baghdad teaching hospital. Mode of presentation, age, sex, predisposing conditions, diagnostic modalities, operative findings and type of operative procedure performed were all studied and analyzed. There were (24) males and (26) females. Male: female ratio about 0.9: 1, the commonest age groups presented were first between 25 - 35 years (14 patients) and second between 46 - 55 years (16 patients). The main presentation which is abdominal pain and gastrointestinal bleeding ( 33 cases) was the most lethal one, with a mortality rate of $(64.5 \%)$. The main physical finding was patient with signs of peritoneal irritation (38 patients) which carries lower mortality rate $(21 \%)$, while the highest mortality rate was seen in those having combined features of peritoneal irritation and hemodynamic instability $(87.5 \%)$. The time interval between hospital admission and time of surgery ranged from $(<24-244$ hours $)$ with a mean $=22$ patients have two or more predisposing conditions like hypertension, diabetes, ischemic heart disease and atrial fibrillation. Different types of diagnostic modalities were performed, CT scan carries high diagnostic accuracy rate $(100 \%)$. The commonest operative findings were focal segmental ischemia (38 cases) explaining the most frequent operative procedure performed which is resection with end to end anastomosis (32 cases). The only significant risk factors affecting the outcome of patients in our study were age (old age group), delayed interval between hospital admission and onset of surgery, late presentation (gastrointestinal bleeding), physical findings (combination of signs of peritoneal irritation and hemodynamic instability), number of predisposing conditions (more than two), operative finding (extensive bowel ischemia) and operative procedure performed (resection with end to end anastomosis).

Keywords: Anastomosis, ischemia, bowel

\section{Introduction}

Occlusion of the mesenteric vessels is apt to be regarded as one of those conditions of which the diagnosis is impossible, the prognosis hopeless, and the treatment almost useless" (Cokkinis, 1926). AMI is a syndrome in which inadequate blood flow through the mesenteric circulation causes ischemia and eventual gangrene of the bowel wall ${ }^{[1]}$.

Anatomy: Typically, the celiac artery (CA) supplies the foregut, hepatobiliary system, and spleen; the superior mesenteric artery (SMA) supplies the midgut (ie, small intestine and proximal mid colon); and the inferior mesenteric artery (IMA) supplies the hindgut (ie, distal Colon and rectum), but multiple anatomic variants are observed. Venous drainage is through the superior mesenteric vein (SMV), which joins the portal vein ${ }^{[2]}$.

Pathophysiology: Insufficient blood perfusion to the small bowel and colon may result from arterial occlusion by embolus or thrombosis (AMAE or AMAT), thrombosis of the venous system (MVT), or nonocclusive processes such as vasospasm or low cardiac output (NOMI). Embolic phenomena account for approximately $50 \%$ of all cases, arterial thrombosis for about $25 \%$, NOMI for roughly $20 \%$, and MVT for less than $10 \%$. Hemorrhagic infarction is the common pathologic pathway whether the occlusion is arterial or venous. Injury severity is inversely proportional to the mesenteric blood flow and is influenced by the number of vessels involved, systemic mean pressure, duration of ischemia, and collateral circulation. The superior mesenteric vessels are involved more frequently than the inferior mesenteric vessels, with blockage of the latter often being silent because of better collateral circulation. Damage to the affected bowel portion may range from reversible ischemia to transmural infarction with necrosis and perforation.
Correspondence

Dr. Labed Saadallah Abdulkareem Fallujah Teaching Hospital, Iraq 
The injury is complicated by reactive vasospasm in the SMA region after the initial occlusion. Arterial insufficiency causes tissue hypoxia, leading to initial bowel wall spasm. This leads to gut emptying by vomiting or diarrhea. Mucosal sloughing may cause bleeding into the gastrointestinal tract. At this stage, little abdominal tenderness is usually present, producing the classic intense visceral pain disproportionate to physical examination findings. The mucosal barrier becomes disrupted as the ischemia persists, and bacteria, toxins, and vasoactive substances are released into the systemic circulation. This can cause death from septic shock, cardiac failure, or multisystem organ failure before bowel necrosis actually occurs. As hypoxic damage worsens, the bowel wall becomes edematous and cyanotic. Fluid is released into the peritoneal cavity, explaining the serosanguinous fluid sometimes recovered by diagnostic peritoneal lavage. Bowel necrosis can occur in 8-12 hours from the onset of symptoms. Transmural necrosis leads to peritoneal signs and heralds a much worse prognosis ${ }^{[3]}$

\section{Clinical presentation}

Early identification of AMI required a high index of suspicion for those patients who have significant risk factors associated with the disease. AMI occur most frequently in patients $>50$ years of age who have chronic heart disease cardiac arrhythmia especially atrial fibrillation, recent MI, or hypotension due to burn, pancreatitis or haemorrhage. Previous or synchronous arterial emboli increase the likelihood of an acute superior mesenteric artery embolism.

The development of sudden abdominal pain in patients with any of these risk factors should suggest the diagnosis of AMI. Sudden severe abdominal pain accompanied by rapid and often forceful bowel evacuation especially with minimal or no abdominal signs strongly suggest acute arterial occlusion in the mesenteric circulation. Unexplained abdominal distension or gastrointestinal bleeding may be the only indication of AMI especially in non-occlusive disease, since pain is absent in up to $25 \%$ of these patients ${ }^{[4]}$. Patients surviving cardiopulmonary resuscitation who develop culture proven bacteraemia and diarrhea without abdominal pain should be suspected of having NOMI ${ }^{[5]}$. Distension while absent early in the coarse of mesenteric ischemia is often the first sign of impending intestinal infarction. The stool contained occult blood in $75 \%$ and this bleeding may proceed any other symptoms of ischemia. As infarction develop increasing tenderness, rebound tenderness and muscle guarding reflect the progressive loss of intestinal viability and the presence of transmural gangrene, significant abdominal finding strongly indicate the presence of infracted bowel. Nausea, vomiting, hematochezia, haematemesis, massive abdominal distension, back pain and shock are other late signs that indicate compromise of bowel viability ${ }^{[4]}$.

\section{Diagnosis}

\section{Laboratory studies}

- Leukocytosis exceeding 15000 cell $/ \mathrm{mm}^{3}$ occur in approximately $75 \%$ of patients with AMI.

- Serum amylase levels are moderately elevated in over $50 \%$ of patients but this finding is nonspecific.

- Metabolic acidosis is observed late in the disease coarse ${ }^{[6]}$.

\section{Imaging studies}

- Plain abdominal film findings on plain film are usually normal before infarction, as disease progress pattern of adynamic ileus, gasless abdomen or small bowel pseudoobstruction can be noted. Late in the coarse of the disease "pinky printing" can suggest the diagnosis of AMI, less commonly isolated thumb printing of right colon may be the only indication of AMI. Rare findings include: pneumatosis or gas in portal in the venous system.

- Duplex scanning has been of some value in the diagnosis of AMI.

- CT scan helps to evaluate AMI and exclude other causes of abdominal pain. It may show pneumatosis intestinalis, portal vein gas, bowel wall and or mesenteric edema.

- Angiography selective angiography is the main stay of diagnosis and essential treatment of occlusive and nonocclusive AMI ${ }^{[7]}$

- Laparoscopy may be useful for patients whose clinical status preclude angiography however, laparoscopic examination of the bowel is limited to the serosal surface making it unreliable for diagnosing early mucosal necrosis at a time the serosa still appear relatively normal ${ }^{[8]}$.

\section{Treatment}

Initially, the primary physician should focus on resuscitation of the patient, stabilization of cardiac function, and initiation of antibiotic therapy with broad-spectrum coverage. Medications that have vasoconstrictive effects should be discontinued. If there is evidence of peritonitis, a surgical consultation and laparotomy are indicated. A "second look" laparotomy, 24 to 48 hours after the first surgery, is performed by many surgeons when the viability of the nonresected bowel is in doubt. For angiographically diagnosed SMA emboli, treatment options to restore intestinal blood flow include surgical revascularization, intra-arterial thrombolysis, intra-arterial vasodilatation, and systemic anticoagulation. If peritonitis is present, surgical embolectomy with resection of infarcted bowel is indicated. In the absence of peritonitis, surgical embolectomy is the treatment of choice for major emboli. Thrombolytic therapy (streptokinase, Urokinase, or recombinant tissue plasminogen activator) has been reported to be successful in individual cases and in small series of patients. Thrombolysis has a higher probability of success when performed within 12 hours of symptom onset. Following embolectomy, infusion of the vasodilator Papaverine has been recommended by many investigators to prevent further ischemia due to vasospasm of the mesenteric arteries. Postoperative anticoagulation is used to prevent recurrence. Surgical revascularization is indicated for treatment of acute mesenteric thrombosis. Thrombectomy alone does not provide long-term relief because thrombogenicity atherosclerotic plaques remain in the vessel. Although successful therapy with thrombolytic infusion and angioplasty has been reported in a subset of patients, this approach is not yet the standard of care. Patients with NOMI but without intestinal infarction have been successfully treated with intra-arterial infusion of Papaverine. Anticoagulation alone is the mainstay of treatment for patients with SMVT who do not have evidence of infarcted bowel ${ }^{[9]}$.

\section{Aim of the study}

1. To highlight the problem of acute mesenteric ischemia.

2. To analyze the clinical presentation of acute mesenteric ischemia as surgical emergency.

3. To evaluate the validity of available diagnostic procedure in diagnosing acute mesenteric ischemia. 
4. To assess the risk factors such as hypertension, ischemic heart, atrial fibrillation....

5. To assess the best surgical procedure.

6. Conclusion and recommendation to improve the outcome.

\section{Patient's and methods}

The records of 50 patients who were admitted to the surgical causality department of Baghdad Teaching Hospital who were diagnosed at laparotomy as having mesenteric ischemia in the period between 1995 - 2005 were reviewed. The clinical records of these patients were reviewed with respect to clinical presentation, sex, age, past-medical history, time interval between presentation and treatment, operative procedure performed and outcome. Certain risk factors for death were analyzed statistically.

\section{Results and Tables}

The records of a total number of 50 patients with mesenteric ischemia were reviewed. There were 24 men $(48 \%)$ and 26 women $(52 \%)$, with male: female ratio $=0.9$ : 1 . (Table 1 ) Although the most common age groups affected by the disease in our study sample were first between 25 - 35 years (14 patients) and second between 46 - 55 years (16 patients), but the mortality rate was increasing gradually with increasing age to reach $100 \%$ at age group 66-75 years. (Table 2) The most lethal presentation was gastrointestinal bleeding in 33 patients (mortality rate $=63.6 \%$ ), while those presenting with a clinical picture of abdominal pain, vomiting and diarrhea followed by constipation was found in 17 patients with a mortality rate of $17.6 \%$. (Table 3 ) Table 4 - showed that the victims of patients in our study sample were most prevalent in those with physical findings of hemodynamic instability and those with a combination of hemodynamic instability and peritoneal signs with a mortality rate of $70 \%, 80 \%$ respectively. While the mortality rate in those presenting with peritoneal signs was significantly less $37 \%$. The time interval between hospital admission and the time of operation ranged from $(<24$ hours 244 hours), with progressive increase in mortality rate with delay onset of surgical intervention.(table 5) A review of systems and past-medical history looking for predisposing factors of mesenteric ischemia was performed, 22 patients had two or more predisposing factors \& constitute the higher mortality groups $(71.4 \%)$, while the mortality rate are decreased in patients with single predisposing factors until it reach $12.5 \%$ in patient without apparently underlying predisposing condition.
(Table 6)

In respect to the different diagnostic modalities used, plain abdominal radiography and WBC's count which were performed for all patient, they were positive in 27 patient with non-specific radiographic findings in term of ileus and evidence of small bowel obstruction and in 35 patients with WBC's count $>20 \mathrm{x}$ $10^{9}$ respectively. While the positive findings suggestive of mesenteric ischemia was present in 23 out of 41 patients for whom serum amylase was done and in 15 out of 45 patients for those undergo ultrasound examinations. Although CT scan was done to only 10 patients owing to the difficulties in obtaining the test early in our country, it was positive in all of them. (Table 7) As a result of the delayed onset of surgical intervention and inability to do angiography for the suspected cases, all of the patients in our study have gangrenous bowel with variable length of involvement. The most common operative finding was focal segmental ischemia in 38 patients and this explaining the most frequent surgical procedure performed which is surgical resection with end to end anastomosis (33 patients), but unfortunately carried higher mortality rate $60.6 \%$, while surgical resection with exteriorization of the two segment was done to 16 patients with lower mortality rate 18.7 $\%$.Unfortunately open and closed technique (explorative laparotomy) was done to one patient who has extensive bowel gangrene with evidence of systemic sepsis with $100 \%$ mortality rate. (Table 8 and 9) Vascular procedures were not carried out in any. No planned second-look procedures were carried out

Table 1: Number and percentage of patients according to sex distribution

\begin{tabular}{|c|c|c|}
\hline Sex & No. of patients & Percent \\
\hline Male & 24 & $48 \%$ \\
\hline Female & 26 & $52 \%$ \\
\hline Total & 50 & $100 \%$ \\
\hline
\end{tabular}

Table 2: Distribution of patients according to age groups and the mortality rate for each group

\begin{tabular}{|c|c|c|}
\hline Age group & No. of patient $\mathbf{( \% )}$ & No. of death $\mathbf{( \% )}$ \\
\hline $25-35$ years & $14(28 \%)$ & $5(35.7 \%)$ \\
\hline $36-45$ years & $4(8 \%)$ & $1(25 \%)$ \\
\hline $46-55$ years & $16(32 \%)$ & $6(37.5 \%)$ \\
\hline $56-65$ years & $8(16 \%)$ & $4(50 \%)$ \\
\hline $66-75$ years & $8(16 \%)$ & $8(100 \%)$ \\
\hline Total & $50(100 \%)$ & $24(48 \%)$ \\
\hline
\end{tabular}

Table 3: Number of patients and number of death according to clinical presentation

\begin{tabular}{|c|c|c|}
\hline Mode of presentation & No. of patient (\%) & No. of death (\%) \\
\hline Abdominal pain with gastrointestinal bleeding & $33(66 \%)$ & $21(63.6 \%)$ \\
\hline Abdominal pain, vomiting, diarrhea followed by constipation & $17(34 \%)$ & $3(17.6 \%)$ \\
\hline Total & 50 & 24 \\
\hline
\end{tabular}

Table 4: Number of patients and number of death according to the physical findings

\begin{tabular}{|c|c|c|}
\hline Physical findings & No. of patients (\%) & No. of death (\%) \\
\hline Peritoneal signs & $35(70 \%)$ & $13(37 \%)$ \\
\hline Hemodynamic instability & $10(20 \%)$ & $7(70 \%)$ \\
\hline Combined features & $5(10 \%)$ & $4(80 \%)$ \\
\hline
\end{tabular}

Table 5: Number of patients and number of death according to the time interval between hospital admission and time of surgery

\begin{tabular}{|c|c|c|}
\hline Time (hours) & No. of patients (\%) & No. of death (\%) \\
\hline$<24$ & $4(8 \%)$ & $1(25 \%)$ \\
\hline $24-72$ & $14(28 \%)$ & $4(28.6 \%)$ \\
\hline $72-196$ & $24(48 \%)$ & $13(54 \%)$ \\
\hline $196-244$ & $8(16 \%)$ & $6(75 \%)$ \\
\hline
\end{tabular}


International Journal of Surgery Science

Total 
Table 6: Number of patients and number of death according to the type and number of risk factors present

\begin{tabular}{|c|c|c|}
\hline Risk factor & No. of patients (\%) & No. of death (\%) \\
\hline Hypertension, diabetes and ischemic heart disease & $14(28 \%)$ & $10(71.4 \%)$ \\
\hline Atrial fibrillation and ischemic heart disease & $8(16 \%)$ & $5(62.5 \%)$ \\
\hline History of coagulation disorders & $8(16 \%)$ & $4(50 \%)$ \\
\hline Hypertension & $4(8 \%)$ & $2(50 \%)$ \\
\hline Post-partum & $8(16 \%)$ & $2(25 \%)$ \\
\hline No risk factors & $8(16 \%)$ & $1(12.5 \%)$ \\
\hline Total & 50 & 24 \\
\hline
\end{tabular}

Table 7: Types of diagnostic modalities

\begin{tabular}{|c|c|c|}
\hline Diagnostic procedure & No. of patients Undergo the test (\%) & No. of patients with Positive findings (\%) \\
\hline WBC's count & $50(100 \%)$ & $35(70 \%)$ \\
\hline Serum amylase & $41(82 \%)$ & $23(80 \%)$ \\
\hline Plain abdominal film & $50(100 \%)$ & $27(64 \%)$ \\
\hline Abdominal ultrasound & $45(90 \%)$ & $15(32 \%)$ \\
\hline CT scan & $10(20 \%)$ & $10(100 \%)$ \\
\hline
\end{tabular}

Table 8: Relation of the operative findings with the number of death

\begin{tabular}{|c|c|c|}
\hline Operative finding & No. of patients (\%) & No. of death (\%) \\
\hline Extensive bowel ischemia & $12(24 \%)$ & $11(91.7 \%)$ \\
\hline Focal segmental ischemia & $38(76 \%)$ & $13(34.2 \%)$ \\
\hline Total & 50 & 24 \\
\hline
\end{tabular}

Table 9: Relation of type of operative procedure with the number of death

\begin{tabular}{|c|c|c|}
\hline Type of operative procedure & No. of patient (\%) & No. of death (\%) \\
\hline Resection with end to end anastomosis & $33(66 \%)$ & $20(60.6 \%)$ \\
\hline Resection with exteriorization of bowel segment & $16(32 \%)$ & $3(18.7 \%)$ \\
\hline Re-vascularization & 0 & 0 \\
\hline Open and closed technique & $1(2 \%)$ & $1(100 \%)$ \\
\hline
\end{tabular}

\section{Discussion}

In the US: Currently, the overall prevalence of AMI is $0.1 \%$ of all hospital admissions; this may be expected to rise as the population ages ${ }^{[1]}$. Internationally: Rates of AMI have not been demonstrated to be significantly different outside the United States. However, because it is primarily a disease of older individuals, rates are probably lower in countries whose populations have shorter life expectancy ${ }^{[1]}$. In relation to male: female ratio, we found that it is about $0.9: 1$ which is in agreement with other studies which showed that no overall sex preference exist for mesenteric ischemia ${ }^{[1]}$. As seen in this study the incidence of the disease was high in two age groups first between $25-35$ years and second between $46-55$ years. While Chat Dang stated that 'AMI is frequently considered a disease of people older than 50 years' ${ }^{\text {[1] }}$, This could be attributed to the fact that not very many people in our country reach this age group who have chronic heart disease and long standing congestive heart failure especially those poorly controlled with diuretics or digitalis ${ }^{[6]}$. In comparison to other studies ${ }^{[10-12]}, 66 \%$ of the patients in our study sample were presented as abdominal pain with gastrointestinal bleeding, while the remaining $34 \%$ were presented with a clinical picture of abdominal pain, anorexia, vomiting and diarrhea followed by constipation. From our data it is apparent that the majority of patients $(70 \%)$ were presented with a physical finding of peritonitis and $20 \%$ with hemodynamic instability, this result agreed with other study conducted by Edwards MS et al. which described that $64 \%$ of patients were presented with peritonitis and $30 \%$ with hypotension ${ }^{[13]}$. Despite our effort to diagnose the disease early, the time interval between hospital admission and the time of operation in our study sample ranged from < $24-244$ hours (48 $\%$ of patients studied ranged from $72-196$ hours), which is in agreement with Divino CM et al. in view of the time to surgery which was ranged from 3 hours to 7 days after admission in his study sample ${ }^{[12]}$. It is a well-known fact that there are predisposing factors responsible for the disease as $84 \%$ of the patients in this study had one or more predisposing factors responsible for the embolic or thrombotic events such as atrial fibrillation, ischemic heart disease, hypertension, diabetes mellitus and history of coagulation disorders. This is similar to previous publication documenting that $50 \%$ of cases of acute mesenteric ischemia occur as a result of left ventricular infarction or atrial fibrillation, $25 \%$ of cases is the end result of atherosclerosis and $5 \%$ of cases is associated with a hypercoagulability states ${ }^{[14]}$. Although our findings of an elevation of WBC's count (> $\left.20 \times 10^{9}\right)$ and serum amylase which were present in $70 \%$ and $80 \%$ of patients studied respectively, are in accord with other study conducted by Chat Dang where elevation of both WBC's count and serum amylase were present in more than $50 \%$ of cases ${ }^{[1]}$, but these findings are nonspecific. We agree with those who suggest that laboratory studies are not helpful in diagnosing mesenteric ischemia ${ }^{[1]}$. Currently, no serum marker is sensitive or specific enough to establish or exclude the diagnosis of mesenteric ischemia. Waiting for laboratory results should not delay radiographic studies if serious suspicion of mesenteric ischemia exists ${ }^{[1]}$. Findings on plain films of the abdomen often are normal in the presence of mesenteric ischemia. In the present study, $64 \%$ of our patients showed non-specific signs of mesenteric ischemia in term of ileus, small bowel obstruction, edematous/thickened bowel walls, and paucity of gas in the intestines, our result agreed with that conducted by Bottger $\mathrm{T}$ where the $\mathrm{X}$-ray of the abdomen shows signs of an ileus in more than half of the patients, while Andersson $\mathrm{R}$ stated that 'The most frequently 
used investigation was plain abdominal X-ray with a positive finding in only 1/3' [13, 16]. More specific signs, such as pneumatosis intestinalis, ie, submucosal gas; thumbprinting of bowel wall; and portal vein gas, are late findings ${ }^{[1]}$. In one study of 23 cases of bowel infarction, $30 \%$ of the patients demonstrated focally edematous bowel wall (thumbprinting) and/or pneumatosis intestinalis, while we couldn't demonstrate such findings in our study ${ }^{[1]}$. However, plain films are warranted to exclude identifiable causes of abdominal pain such as perforated viscus with free intraperitoneal air ${ }^{[1]}$. In the study of 145 patients reported by Czerny $\mathrm{M}$, the abdominal sonography led to the correct diagnostic assessment in 52 patients $(=35.8 \%)$, result nearly closer to our abdominal ultrasound reports which were positive in 15 patients $(33 \%)$ out of 45 patients underwent the test ${ }^{[17]}$. Ultrasound is considered a second-line study for mesenteric ischemia. It is often less useful in the presence of dilated fluid-filled loops of bowel ${ }^{[1]}$. Chat Dung stated that 'Duplex sonography studies are highly specific (92-100\%) but not as sensitive (70-89\%) compared to angiography'. In fact Duplex sonography was not used in the evaluation of our studied patients ${ }^{[1]}$. Recent interests in the diagnosis of mesenteric ischemia has focused on the role of vascular enhanced CT scan ${ }^{[18,19]}$. We agree with Rhee RY and Morasch MD in that CT scan offers significant information and is the most sensitive diagnostic test as Computed tomography (CT) was considered diagnostic in $10(100 \%)$ of 10 patients who underwent the test in our study sample ${ }^{[20,21]}$. Spiral CT (ideally using a Multislice technique) can accurately demonstrate morphology of the arterial and venous mesenteric vessels, changes in the bowel wall, and additional mesenteric or peritoneal findings. Therefore, CT has the potential for diagnosis of mesenteric ischemia on a pathological basis. Furthermore, CT is successfully used to confirm or exclude most other causes of acute abdominal conditions ${ }^{[19]}$. Our study was restricted to patients with frank gangrene of the bowel with variable length of involvement. The commonest operative finding in our series was focal segmental ischemia $(76 \%)$, this is similar to what was published by Scott J. and Ronald N. describing that $95 \%$ of their patient had segmental involvement of the jejunum and / or ileum ${ }^{[4]}$. While vascular procedures were not carried out in any, intestinal resection with end to end anastomosis was the most frequent operative procedures performed in our study sample $(68 \%)$, resection with exteriorization of the bowel segments was done in $(30 \%)$ of cases and open and closed technique (exploratory laparotomy) was done to one patient only (2\%). These results concede with several other series [22, 23], where resection with end to end anastomosis was the most frequent operative procedure performed. Although no patient underwent a second-look operation in our study, it has been suggested that if extensive portions of the bowel are involved, only the clearly necrotic bowel is resected and a planned re-exploration (secondlook operation) is performed within 12 to 24 hours. The decision to perform a second-look operation is made during the initial celiotomy, if major portions or multiple segments of intestine are of equivocal viability. The purpose of the second-look celiotomy as apposed by Shaw is "not just to allow a clear definition between dead and live bowel to take place, but also to allow time for the institution of supportive measures which may render more of the bowel viable." ${ }^{[24]}$ Such measures may include optimizing cardiac output, SMA infusion with Papaverine, antibiotic therapy, and anticoagulant therapy. Once made, the decision to perform a second-look operation is inviolate and must be done irrespective of the clinical course of the patient. If it is planned, anastomoses need not be made until the time of the re-exploration. Only $18 \%$ of second-look procedures have been shown to contribute to patient survival. ${ }^{(4)}$ We verified the factors that affect the outcome of our patients which include:- age group, mode of presentation, history of co morbid diseases, time interval to surgery, operative finding and type of operative procedure performed. We confirm the findings of Paes E et al. who reported that old age ( 70.6 years in average) and a long time interval between onset of symptoms and therapy $(25.8 \mathrm{~h}$ in average) are responsible for the still high operative mortality $\left(52.9 \%\right.$ in the reported series) ${ }^{[25]}$. The mortality rate is higher in elderly patients than in younger patients which are mainly due to delayed surgery in the elderly group. If surgery is performed early the survival rate increases independent of age. The most important prognostic factor and the only factor that can be influenced by the surgeon is the time interval between onset of symptoms and surgery ${ }^{[26]}$. While Urayama $\mathrm{H}$ et al. stated that mortality was higher in patients with advanced peritonitis [27], we found that it is higher in patients with combinations of hemodynamic instability and peritonitis (87. 5 $\%)$. This is attributed to the shift of intravascular volume into the bowel wall causes severe hem concentration and hypovolemic shock. Vasoactive mediators and bacterial endotoxins are released from the ischemic bowel into the peritoneal cavity and absorbed into the general circulation, causing a variety of physiological effects, including cardiac depression, septic shock, and acute renal failure. These effects may contribute to the death of the patient even before there is complete necrosis of the bowel wall ${ }^{[4]}$. It has been postulated in one study that Mortality rate of all patients was $70.3 \%$ and showed in further analysis significant differences in age, etiology of mesenteric ischemia, the length of ischemic and operatively resected gut ${ }^{[28]}$, this is supported by the results of this study which demonstrate high mortality rates with the increasing number of predisposing conditions and length of ischemic and resected bowel, 91.6 and $84 \%$ respectively. In spite of what was reported by Warshauer $\mathrm{D} \mathrm{M}$ et al. that 'no correlation was noted between predisposing factors and outcome' [29], the result of this study has shown that with the ever increasing number of predisposing factors, the mortality rate increases as well. This is not unexpected given the fact that these concomitant medical illnesses added worse to the emergency operations performed for those patients. In our study, we found that the mortality rate was high in those presenting with abdominal pain and gastrointestinal bleeding (63.6\%), this can be explained by the fact that gastrointestinal bleeding is considered as a late presentation of patient with mesenteric ischemia with all the sequel of tissue hypoxia and reperfusion injury.

\section{Conclusion}

The prevalence of mesenteric ischemia internationally is lower than that in United State because it is primarily a disease of older individuals, rates are probably lower in countries whose populations have shorter life expectancy. Those who discovered to have mesenteric ischemia were most commonly presented with abdominal pain and gastrointestinal bleeding with a physical finding of peritoneal irritation. There is no overall sex preponderance of those with mesenteric ischemia. Old age groups have high mortality rates, as well as those presenting with gastrointestinal bleeding and combined signs of peritoneal irritation and hemodynamic instability. $\mathrm{Ct}$ scan has high diagnostic accuracy rate. Most patients with mesenteric ischemia have one or more predisposing conditions responsible for the 
disease and it's high mortality rate. Patients with extensive bowel ischemia have high mortality rate as well as those treated with resection and end to end anastomosis.

\section{Recommendations}

1. AMI is potentially lethal emergency disease, early diagnosis, anticoagulation and appropriate surgical approach is essential to improve the outcome.

2. Smoking cessation, dietary modification and blood pressure control are all required for prevention of atherosclerotic disease. Control of heart arrhythmias is an important preventive measure for AMI.

3. Its important to keep vigilance for AMI in the patient in the hyper coagulation status specially when the symptoms are inconsistent with abdominal signs.

4. AMI must be kept in mind as a possible diagnosis in patients with abdominal pain, predisposing factors are frequently associated and should searched for.

5. Early diagnosis using CT scan, color ultrasound enable immediate anticoagulation and intervention and seems to improve outcome.

6. If resection with end - end anastomosis is the preferred procedure by an operating surgeon we recommend second look procedure to assess the viability of the bowel, otherwise intestinal resection with exteriorization of the resected ends is the preferred option.

\section{References}

1. Chat Dang, Jeff Wade, Burt Cagir et al. Last Updated on Acute Mesenteric Ischemia. Academic Emergency Medicine, 2004.

2. Richard S Snell. The abdominal cavity, Clinical anatomy, Lippincott Williams and Wilkins, Philadelphia. $6^{\text {th }}$ edn, 2000, 218-222.

3. Walter JB, Talbot IC. Walter and Israel General Pathology. Churchill Livingstone. 1996; 7:447-9.

4. Scott J Boley, Ronald N Kaleya. Mesenteric ischemic disorders. Chap. 18 Michael J. Zinner, Symmour I. Schwartz, Harold Ellis et al. Maingot's abdominal operations. Appleton \& Lange, USA. 1997; 10:655-690.

5. Gaussorgues $\mathrm{P}$, Guerugniand $\mathrm{PY}$, Vedrinne JM et al. Bacteremia following cardiac arrest and cardiopulmonary resuscitation. Intensive care med. 1988; 14:575-580.

6. Kenneth Ouriel, Richard M Green. Arterial disease Seymour I. Schwartz, Principles of surgery, McGraw-Hill, New York. 1999; 7:966-967.

7. Smerud MJ, Johnson CD, Stephens DH. Diagnosis of bowel infarction: a comparison of plain films and CT scan in 23 cases. Am J Roenntgenol 1990: 154:99-102.

8. Serreyn RF, Schoofs PR, Baetenns PR et al. laparoscopic diagnosis of mesenteric venous thrombosis. Endoscopy 1986: 18:249-251.

9. Brandt L, Boley S. AGA Technical Review on Intestinal Ischemia. Gastroenterology. 2000; 118:954.

10. Park WM, Gloviczki P, Cherry KJ Jr et al. Contemporary management of acute mesenteric ischemia: Factors associated with survival.J Vasc Surg. 2002; 35(3):445-52.

11. Morasch MD, Ebaugh JL, Chiou AC et al. Mesenteric venous thrombosis: a changing clinical entity. J Vasc Surg. 2001; 34(4):680-4.

12. Divino CM, Park IS, Angel LP et al. A retrospective study of diagnosis and management of mesenteric vein thrombosis Am J Surg. 2001; 181(1):20-3.

13. Edwards MS, Cherr GS, Craven TE et al. Acute occlusive mesenteric ischemia: surgical management and outcomes. Ann Vasc Surg. Epub 2003 Jan 15. 2003; 17(1):72-9.

14. Andrerw A Shelton, Theodore R Schrock, Mark Lane Welton. Small intestine Chap.30, Lawrence W. Way, Gerard M. Dohetry, Current surgical diagnosis and treatment, $11^{\text {th }}$ ed., a LANGE medical book, New York, 2003, 698.

15. Bottger T, Schafer W, Weber W et al. Sensitivity of preoperative diagnosis in mesenteric vascular occlusion, Bildgebung. 1991; 58(4):192-8.

16. Andersson R, Parsson H, Isaksson B et al. Acute intestinal ischemia. A 14-year retrospective investigation. Acta Chir Scand. 1984; 150(3):217-21.

17. Czerny M, Trubel W, Claeys L et al. Acute mesenteric ischemia Zentralbl Chir. 1997; 122(7):538-44.

18. Karen M Horton, Elliot K. Fishman, Multi-Detector Row CT of Mesenteric Ischemia: Can It Be Done? Radio graphics. 2001; 21:1463-1473.

19. Duber C, Wustner M, Diehl SJ et al. Emergency diagnostic imaging in mesenteric ischemia Chirurg. 2003; 74(5):399406.

20. Rhee RY, Gloviczki P, Mendonca CT et al. Mesenteric venous thrombosis: still a lethal disease in the. $1990 \mathrm{~J}$ Vasc Surg. 1994; 20(5):688-97.

21. Morasch MD, Ebaugh JL, Chiou AC et al. Mesenteric venous thrombosis: a changing clinical entity. J Vasc Surg. 2001; 34(4):680-4.

22. Lefrancois C, Derlon A, Le Querrec A et al. Mesentric venous thrombosis. Risk factors, treatment and outcome. An analysis of 18 cases. Ann Fr Anesth Reanim. 1994; 13(2):182-94.

23. Divino CM, Park IS, Angel LP et al. A retrospective study of diagnosis and management of mesenteric vein thrombosis. Am J Surg. 2001; 181(1):20-3.

24. Shaw RS. The "second look" after superior mesenteric arterial embolectomy or reconstruction for mesenteric infarction. In: Ellison EH, Frieser SR, Mulholland JH (eds), Current Surgical Management. Philadelphia, PA: WB Saunders; 1965, 509.

25. Paes E, Vollmar JF, Hutschenreiter S et al. Mesenterial infarct. New aspects of diagnosis and therapy. Chirurgic. 1988; 59(12):828-35.

26. Ritz JP, Runkel N, Berger G et al. Prognostic factors in mesenteric infarct. Zentralbl Chir. 1997; 122(5):332-8.

27. Urayama H, Ohtake H, Kawakami T et al. Acute mesenteric vascular occlusion: analysis of 39 patients. Eur J Surg. 1998; 164(3):195-200.

28. Jonas J, Bottger T. Diagnosis and prognosis of mesenterial infarct. Med Klin (Munich). 1994; 89(2):68-72.

29. Warshauer DM, Lee JK, Mauro MA et al. Superior mesenteric vein thrombosis with radiologically occult cause: a retrospective study of 43 cases. AJR-Am-JRoentgenol. 2001; 177(4):837-41. 